\section{Chasing crystals out of the body: will treat to serum urate target for gout help us get there?}

\author{
Jasvinder A Singh, ${ }^{1,2,3}$ Till Uhlig $^{4,5}$
}

In this issue of the journal, Kiltz et al published the treat-to-target (T2T) recommendations for gout, ${ }^{1}$ based on a systematic literature review (SLR) using standard methodology based on Oxford Center for Evidence-based Medicine system and the AGREE criteria. ${ }^{2} 3$ The expert group developed nine T2T recommendations for gout management.

Key $\mathrm{T} 2 \mathrm{~T}$ recommendations were that serum urate (sUA) should be lowered and maintained at $<6 \mathrm{mg} / \mathrm{dL}(<360 \mu \mathrm{mol} / \mathrm{L})$ in all patients with gout (high-level evidence) and at $<5 \mathrm{mg} / \mathrm{dL}(<300 \mu \mathrm{mol} / \mathrm{L})$ in those with severe gout (including tophi or frequent attacks; expert opinion). Inherent to achieving target sUA was the recommendation that sUA should be measured regularly in patients with gout (moderate-level evidence), so that uratelowering therapy (ULT) dose adjustment can be guided by the sUA level. ${ }^{1}$ An updated literature review of T2T strategies in gout identified is a lack of randomised trials that have tested the T2T strategy for gout versus other approaches. The panel also agreed that an appropriate strategic approach to treatment of asymptomatic hyperuricaemia needs to be developed, but lack of evidence prevented the group from making a recommendation regarding a treatment target different than the one recommended for gout.

These recommendations and gout treatment guidelines ${ }^{4-6}$ already support a T2T tactic in gout with a goal of sUA target of $<6 \mathrm{mg} / \mathrm{dL}(<360 \mu \mathrm{mol} / \mathrm{L})$ in all patients with gout and $<5 \mathrm{mg} / \mathrm{dL}(<300 \mu \mathrm{mol} / \mathrm{L})$ in patients with tophaceous or severe gout. Thus, the T2T recommendations for gout in this article 1 are consistent with the

\footnotetext{
${ }^{1}$ Birmingham VA Medical Center, Birmingham, Alabama, USA; ${ }^{2}$ Department of Medicine, School of Medicine, University of Alabama, Birmingham, Alabama, USA; ${ }^{3}$ Division of Epidemiology at the School of Public Health, University of Alabama, Birmingham, Alabama, USA; ${ }^{4}$ National Advisory Unit on Rehabilitation in Rheumatology, Department of Rheumatology, Diakonhjemmet Hospital, Oslo, Norway; ${ }^{5}$ Faculty of Medicine, University of Oslo, Oslo, Norway

Correspondence to Dr Jasvinder A Singh, University of Alabama, Faculty Office Tower 805B, 510 20th Street S, Birmingham, AL 35294, USA; Jasvinder.md@gmail.com
}

previous recommendations/guidelines for the treatment of gout. ${ }^{4-6}$

Although consisting primarily of rheumatologists, the group ought to be congratulated for putting together a multistakeholder team including an internist, nephrologist, cardiologist and a patient. Inclusion of various stakeholders, and not only gout researchers, allows for a richer discussion that goes beyond only one point of view or belief. This inclusive and thoughtful approach also improves the likelihood that this guidance will be taken seriously by practitioners other than rheumatologists.

Some differences in the perceptions related to ULT dosing and target sUA achievement have been noted by patient and physician characteristics. ${ }^{7} 8$ Previous research has suggested that use of antiinflammatory prophylaxis at the initiation of ULT (to prevent acute gout flares) is more common if the provider of gout care is a rheumatologist versus a nonrheumatologist. ${ }^{7}$ Studies of current practice also show that the achievement of target sUA, a key gout outcome, occurs in a low proportion of patients with gout, ranging from $20 \%{ }^{7}$ to $33 \%{ }^{9}$ to $44 \%{ }^{10}$ in various studies. This is primarily due to the lack of knowledge among providers and patients regarding the importance of ULT dosing and achievement of target sUA. $^{11-14}$ Is the patient more likely to achieve target sUA if the provider of gout care is a rheumatologist? This is an important question to answer since the majority of patients with gout in the USA and worldwide are managed by nonrheumatologists (primary care physicians and other specialists). If a difference exists by provider specialty, new programmes and care paradigms could be designed to improve access to specialty care, train primary care physicians regarding the appropriate use of ULT or have nurse- or pharmacist-led programmes.

A recent UK study has shown that a nurse-led programme in a rheumatology clinic consisting of education, individualised lifestyle advice and appropriate ULT helped $>90 \%$ patients with gout from primary care practices to achieve target sUA $<6 \mathrm{mg} / \mathrm{dL}$ during a 12-month follow-up. ${ }^{15}$ Another randomised trial in
California showed that the success rate of target sUA at 26 weeks was significantly higher in patients who received information by phone through a pharmacist versus usual care. ${ }^{16}$ Thus, care by a specialist and/or targeted programmes that include patient education or intensive frequent monitoring can improve our ability to achieve target sUA in gout.

A T2T approach for any chronic disease could be challenging. Despite many years of educational efforts, clinicians continue to manage gout suboptimally. A comprehensive strategy with regular monitoring for sUA level, adverse events, pain and quality of life of patients may be essential for a successful T2T approach in patients with gout.

\section{THE IMPORTANCE OF SUA TARGET: CHASING THE CRYSTALS}

The target recommended for all patients with gout is $\mathrm{sUA}<6 \mathrm{mg} / \mathrm{dL}$ ( $<5 \mathrm{mg} / \mathrm{dL}$ for tophaceous gout or severe gout with frequent flares), according to the current treatment recommendations and this consensus statement. ${ }^{1}$ This sUA target achievement is based on the saturation point for monosodium urate since achievement of target sUA $<6 \mathrm{mg} / \mathrm{dL}$ has been shown to lead to dissolution of crystals, ${ }^{17}$ as well lower risk of gout flares, tophi and medical care costs. ${ }^{18-21}$ A higher sUA is associated with a higher risk of gout flares, ${ }^{18-21}$ which present with severe pain and reduction of health-related quality of life (HRQoL). ${ }^{22} 23$ This makes sUA a biomarker that has high clinical relevance. This is not surprising since hyperuricaemia is the underlying biological abnormality in gout and pathophysiological links of hyperuricaemia with urate crystals and crystal-associated inflammation have been established. Thus, the T2T strategy with a sUA target of $<6 \mathrm{mg} / \mathrm{dL}(<360 \mu \mathrm{mol} / \mathrm{L})$ recommended in the current publication ${ }^{1}$ may help to achieve desirable short- and long-term outcomes in gout, such as lower gout flare rates, less emergency room visits and better work/at-home productivity. Challenges of implementation of this strategy must be considered since ULT non-adherence and non-persistence are well documented. ${ }^{24} 25$ A T2T strategy is unlikely to succeed in the absence of effective interventions to lower rates of ULT non-adherence and non-persistence.

One might argue that a simple cut-off threshold for sUA is arbitrary, and more research is needed to further support this threshold. However, ample evidence already exists that this sUA threshold is related to improvement of several important outcomes, from patient and societal perspectives. ${ }^{17-21}$ sUA levels differ by 
gender and age in the population. It is possible that a different sUA cut-off/target that is more specific for gender, race or underlying comorbidities may emerge in the future. One study of particular interest in this field is the Stop Gout Flare study funded by the US Department of Veterans Affairs (VA), a trial that is about to start enrolling. This 4-year randomised, double-blinded, placebo-controlled noninferiority trial will assess the comparative effectiveness of allopurinol with febuxostat in patients with gout and hyperuricaemia (sUA $>6.8 \mathrm{mg} / \mathrm{dL}$ ), using appropriately titrated doses and a T2T approach to achieve sUA goal of $<6 \mathrm{mg} / \mathrm{dL}^{26}$ While the study will not compare a T2T approach to usual care, it will compare the two most commonly used ULTs and will fill an important knowledge gap regarding the real-world comparative effectiveness of the two most used used ULTs in gout.

The deposition of urate crystal in joints and tendons occurs very early during disease course in gout ${ }^{27}$ and tophi are an important outcome in gout. Just as we are concerned about radiographic damage in rheumatoid arthritis, an aim in gout treatment is to show that urate crystal deposits resolve by a T2T approach. ${ }^{28} \mathrm{~A}$ Norwegian observational study is underway to examine the ultrasonography and dual energy CT features of patients with gout undergoing ULT using a T2T approach with monthly evaluations with adjustment of dosage if the sUA target is not yet achieved.

Cardiovascular outcomes are more frequent in several inflammatory rheumatic conditions. Patients with gout frequently have cardiovascular and renal comorbidities. ${ }^{29}$ With an improved understanding of multisystem effects of hyperuricaemia on heart and kidneys, evidence is emerging for a beneficial effect of ULT on cardiovascular and renal outcomes, ${ }^{30-32}$ even in patients without definite gout. Several ongoing studies will address these questions regarding additional nonmusculoskeletal benefits of ULT. ${ }^{33} 34$ One important knowledge gap that also needs to be addressed is whether reaching target sUA will prevent gout flares and ameliorate the cardiovascular and renal outcomes.

\section{COULD THERE BE UNANTICIPATED HARMS OF T2T APPROACH IN GOUT?}

Even the most promising treatment approaches need careful study. This applies to T2T approach in gout as well. The most obvious potential harm of a T2T with an aggressive ULT titration strategy might be more gout flares in the first few months of ULT initiation. Suboptimal treatment with ULT can also lead to more flares. How many more gout flares during the first weeks or months would T2T versus usual care yield? Could these additional gout flares with T2T in the first few months of ULT therapy be prevented with the guideline-recommended concomitant anti-inflammatory prophylaxis with colchicine or non-steroidal antiinflammatory drugs? ${ }^{4-6}$ We do not have definitive answers to these questions, although observational data suggest that prophylaxis is likely effective. Unanticipated challenges and harms might emerge with a widespread implementation of T2T strategy. Therefore, we need welldesigned prospective studies and trials that can address the balance of benefits, potential harms (flares and other unanticipated harms) and patient burden (cost and frequent visits) for a T2T approach versus usual care in gout. An assessment of preferences of patients and additional burden versus benefits of patients associated with this approach become key considerations for implementation of this approach.

Potential harms would also be an issue if we consider treating patients after their first gout attack, as per the updated European League Against Rheumatism gout recommendations ${ }^{4}$ and the Swedish drug administration. ${ }^{35}$ If we become more aggressive in the treatment of gout at an early stage of the disease with a goal of preventing its future consequences, then we need to study benefit/harms/cost balance of an aggressive approach compared with delaying the initiation of treatment with ULT

\section{RESEARCH GAPS}

Despite a tremendous growth in gout research in the recent decades, several important questions remain. We raised these questions throughout this editorial as knowledge gaps that need to be addressed with future research. Additional key research questions are: What is the risk-benefit-cost balance of $\mathrm{T} 2 \mathrm{~T}$ versus usual care approach in gout? Does this balance vary by the severity of gout, patient characteristics, comorbidity load and by the type of ULT (generic such as allopurinol vs febuxostat, a non-generic more expensive drug)? Is T2T an initial goal or a life-long target? Should T2T definition incorporate patient-reported (PROs) and/or patient-relevant outcomes? Once sUA target is achieved, how frequently should sUA be monitored? Can PROs be surrogates for sUA target once $\mathrm{T} 2 \mathrm{~T}$ approach has succeeded?

\section{THE TAKE HOME MESSAGE}

This article highlights not only the recommendations regarding $\mathrm{T} 2 \mathrm{~T}$ in gout, but also the evidence and evidence gaps in this area, and the importance of sUA monitoring and ULT dosing in patients with gout. For far too long, patients with gout have not been treated optimally ${ }^{7} 25$ despite the availability of effective and safe ULTs, including the traditional ULTs such as allopurinol, uricosurics (probenecid, benzbromarone) and new additions including febuxostat, pegloticase and lesinurad. It is about time that we, healthcare providers, learn to use the already available therapies for gout effectively, practice evidence-based medicine and discard myths and wrong perceptions related to ULT dosing and rare associated harms. This will give gout the respect it deserves. It is painful to see that patients with gout continuing to suffer due to suboptimal management of their illness despite all the therapeutic options we have at our disposal, most of which are widely available as generic, low-cost drugs worldwide. We, as healthcare providers, need to treat gout appropriately and reduce its negative impact on quality of life of our patients. If we do, more patients with gout will experience disease remission and enjoy optimal quality of life and functional ability as their counterparts without gout.

Funding JAS is supported by the resources and the use of facilities at the VA Medical Center at Birmingham, Alabama, USA.

Competing interests JAS has received research grants from Takeda and Savient and consultant fees from Savient, Takeda, Regeneron, Merz, Iroko, Bioiberica, Crealta and Allergan pharmaceuticals, WebMD, UBM LLC and the American College of Rheumatology. JAS serves as the principal investigator for an investigator-initiated study funded by Horizon pharmaceuticals through a grant to DINORA, a 501 (c) (3) entity. JAS is a member of the executive of OMERACT, an organisation that develops outcome measures in rheumatology and receives arms-length funding from 36 companies; a member of the American College of Rheumatology's (ACR) Annual Meeting Planning Committee (AMPC); Chair of the ACR Meet-the-Professor, Workshop and Study Group Subcommittee and a member of the Veterans Affairs Rheumatology Field Advisory Committee. TU has received honorarium or consultant fees from AstraZeneca, Novartis and Sobi.

Provenance and peer review Commissioned; externally peer reviewed.

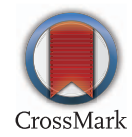

To cite Singh JA, Uhlig T. Ann Rheum Dis 2017;76:629-631.

Received 27 September 2016

Revised 12 November 2016

Accepted 3 December 2016

Published Online First 26 December 2016 


\section{CLinked}

http://dx.doi.org/10.1136/annrheumdis-2016209467

Ann Rheum Dis 2017;76:629-631

doi:10.1136/annrheumdis-2016-210436

\section{REFERENCES}

1 Kiltz U, Smolen J, Bardin T, et al. Treat-to-target (T2T)

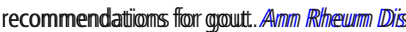
2017:76:632-8.

2 Brouwers MC, Kho ME, Browman GP, et al. AGREE II: advancing guideline development, reporting and evaluation in health care. J Clin Epidemiol 2010:63:1308-11.

3 van der Heijde D, Aletaha D, Carmona L, et al. 2014 Update of the EULAR standardised operating procedures for EULAR-endorsed recommendations. Ann Rheum Dis 2015:74:8-13.

4 Richette P, Doherty M, Pascual E, et al. 2016 updated EULAR evidence-based recommendations for the management of gout. Ann Rheum Dis 2016;76:29-42.

5 Zhang W, Doherty M, Bardin T, et al. EULAR evidence based recommendations for gout. Part II: management. Report of a task force of the EULAR Standing Committee for International Clinical Studies Including Therapeutics (ESCISIT). Ann Rheum Dis 2006;65:1312-24.

6 Khanna D, Fitzgerald JD, Khanna PP, et al. 2012 American College of Rheumatology guidelines for management of gout. Part 1: systematic nonpharmacologic and pharmacologic therapeutic approaches to hyperuricemia. Arthritis Care Res (Hoboken) 2012;64:1431-46.

7 Singh JA, Hodges JS, Asch SM. Opportunities for improving medication use and monitoring in gout. Ann Rheum Dis 2009;68:1265-70.

8 Singh JA, Hodges JS, Toscano JP, et al. Quality of care for gout in the US needs improvement. Arthritis Rheum 2007:57:822-9.

9 Dalbeth $N$, House ME, Horne A, et al. Prescription and dosing of urate-lowering therapy, rather than patient behaviours, are the key modifiable factors associated with targeting serum urate in gout. $B M C$ Musculoskelet Disord 2012:13:174.

10 Singh JA, Akhras KS, Shiozawa A. Comparative effectiveness of urate lowering with febuxostat versus allopurinol in gout: analyses from large US managed care cohort. Arthritis Res Ther 2015:17:120.

11 Harrold LR, Mazor KM, Velten S, et al. Patients and providers view gout differently: a qualitative study. Chronic IIIn 2010;6:263-71.

12 Lindsay K, Gow P, Vanderpyl J, et al. The experience and impact of living with gout: a study of men with chronic gout using a qualitative grounded theory approach. J Clin Rheumatol 2011;17:1-6.

13 Singh JA. Challenges faced by patients in gout treatment: a qualitative study. J Clin Rheumatol 2014;20:172-4.

14 Singh JA. Facilitators and barriers to adherence to urate-lowering therapy in African-Americans with gout: a qualitative study. Arthritis Res Ther 2014;16:R82.

15 Rees F, Jenkins W, Doherty M. Patients with gout adhere to curative treatment if informed appropriately: proof-of-concept observational study. Ann Rheum Dis 2013;72:826-30.

16 Goldfien R, Pressman A, Jacobson A, et al. A pharmacist-staffed, virtual gout management clinic for achieving target serum uric acid levels: a randomized clinical trial. Perm J 2016;20:18-23.

17 Pascual E, Sivera F. Time required for disappearance of urate crystals from synovial fluid after successful hypouricaemic treatment relates to the duration of gout. Ann Rheum Dis 2007;66:1056-8

18 Becker MA, Schumacher HR, MacDonald PA, et al. Clinical efficacy and safety of successful longterm urate lowering with febuxostat or allopurinol in subjects with gout. J Rheumatol 2009;36:1273-82

19 Halpern R, Fuldeore MJ, Mody RR, et al. The effect of serum urate on gout flares and their associated costs: an administrative claims analysis. J Clin Rheumatol 2009:15:3-7.

20 Shoji A, Yamanaka $H$, Kamatani $N$. A retrospective study of the relationship between serum urate leve and recurrent attacks of gouty arthritis: evidence for reduction of recurrent gouty arthritis with antihyperuricemic therapy. Arthritis Rheum 2004;51:321-5.

21 Sundy JS, Baraf HS, Yood RA, et al. Efficacy and tolerability of pegloticase for the treatment of chronic gout in patients refractory to conventional treatment: two randomized controlled trials. JAMA 2011;306:711-20.

22 Khanna PP, Nuki G, Bardin T, et al. Tophi and frequent gout flares are associated with impairments to quality of life, productivity, and increased healthcare resource use: results from a cross-sectiona survey. Health Qual Life Outcomes 2012:10:117.

23 Wood R, Fermer S, Ramachandran S, et al. Patients with gout treated with conventional urate-lowering therapy: association with disease control, health-related quality of life, and work productivity. I Rheumatol 2016:43:1897-903.

24 Briesacher BA, Andrade SE, Fouayzi $\mathrm{H}$, et al. Comparison of drug adherence rates among patients with seven different medical conditions. Pharmacotherapy 2008;28:437-43.

25 Sarawate CA, Brewer KK, Yang W, et al. Gout medication treatment patterns and adherence to standards of care from a managed care perspective. Mayo Clin Proc 2006:81:925-34

26 NIH. Gout: Allopurinol vs. Febuxostat. https:// clinicaltrials.gov/ct2/show/record/NCT02579096. Bethesda, Maryland: Clinicaltrials.gov. A service of the U.S. National Institutes of Health, 2016.

27 Dalbeth N, Stamp L. Hyperuricaemia and gout: time for a new staging system? Ann Rheum Dis 2014:73:1598-600.

28 Villaverde V, Rosario MP, Loza E, et al. Systematic review of the value of ultrasound and magnetic resonance musculoskeletal imaging in the evaluation of response to treatment of gout. Reumatol Clin 2014;10:160-3.

29 Zhu Y, Pandya BJ, Choi HK. Comorbidities of gout and hyperuricemia in the US general population: NHANES 2007-2008. Am J Med 2012;125:679-87. e671.

30 Singh JA, Yu S. Allopurinol and the risk of stroke in older adults receiving medicare. BMC Neurol 2016;16:164.

31 Singh JA, Yu S. Allopurinol and the risk of atria fibrillation in the elderly: a study using Medicare data. Ann Rheum Dis 2016;76:72-8.

32 Goicoechea M, de Vinuesa SG, Verdalles U, et al. Effect of allopurinol in chronic kidney disease progression and cardiovascular risk. Clin J Am Soc Nephrol 2010;5:1388-93.

33 Maahs DM, Caramori L, Cherney DZ, et al. Uric acid lowering to prevent kidney function loss in diabetes: the preventing early renal function loss (PERL) allopurinol study. Curr Diab Rep 2013;13:550-9.

$34 \mathrm{Kim}$ HA, Seo YI, Song YW. Four-week effects of allopurinol and febuxostat treatments on blood pressure and serum creatinine level in gouty men. J Korean Med Sci 2014:29:1077-81.

35 Läkemedelsbehandling av giktbehandlingsrekommendation. Information från Läkemedelsverket. p. 23-33. Drug treatment of gout and treatment recommendations. Information for the Swedish Medical Products Agency. https:// lakemedelsverket.se/upload/halso-och-sjukvard/ behandlingsrekommendationer/ Lakemedelsbehandling_av_gikt_ behandlingsrekommendation.pdf. 2016 